\title{
Power-Law Decay of Standing Waves on the Surface of Topological Insulators
}

\author{
Jing Wang, ${ }^{1}$ Wei Li ${ }^{1}$ Peng Cheng, ${ }^{1}$ Canli Song, ${ }^{1}$ Tong Zhang,,${ }^{1,2}$ Peng Deng, ${ }^{1}$ Xi \\ Chen, ${ }^{1, *}$ Xucun $\mathrm{Ma},{ }^{2} \mathrm{Ke} \mathrm{He}^{2}{ }^{2}$ Jin-Feng Jia, ${ }^{1}$ Qi-Kun Xue, ${ }^{1,2}$ and Bang-Fen Zhu ${ }^{1,3, \dagger}$ \\ 1 State Key Laboratory of Low-Dimensional Quantum Physics, \\ and Department of Physics, Tsinghua University, Beijing 100084, China \\ ${ }^{2}$ Institute of Physics, Chinese Academy of Sciences, Beijing 100190, China \\ ${ }^{3}$ The Institute of Advanced Study, Tsinghua University, Beijing 100084, China
}

(Dated: February 3, 2020)

\begin{abstract}
We propose a general theory on the standing waves (quasiparticle interference pattern) caused by the scattering of surface states off step edges in topological insulators, in which the extremal points on the constant energy contour of surface band play the dominant role. Experimentally we image the interference patterns on both $\mathrm{Bi}_{2} \mathrm{Te}_{3}$ and $\mathrm{Bi}_{2} \mathrm{Se}_{3}$ films by measuring the local density of states using a scanning tunneling microscope. The observed decay indices of the standing waves

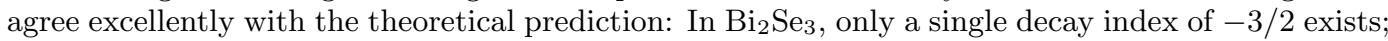
while in $\mathrm{Bi}_{2} \mathrm{Te}_{3}$ with strongly warped surface band, it varies from $-3 / 2$ to $-1 / 2$ and finally to -1 as the energy increases. The $-1 / 2$ decay indicates that the suppression of backscattering due to time-reversal symmetry does not necessarily lead to a spatial decay rate faster than that in the conventional two-dimensional electron system. Our formalism can also explain the characteristic scattering wave vectors of the standing wave caused by non-magnetic impurities on $\mathrm{Bi}_{2} \mathrm{Te}_{3}$.

PACS numbers: 73.20.-r 68.37.Ef 73.43.Cd 72.10.Fk
\end{abstract}

The discovery of topological insulators (TIs) has attracted a great deal of attention [1 12. The threedimensional TIs are characterized by the gapped bulk states and gapless surface states (SSs), which are protected by time-reversal symmetry (TRS) and consist of an odd number of spin-helical Dirac cones. Exotic effects such as Majorana fermions [13, 14] and magnetic monopole [15] are predicted to exist as results of the topological SSs.

The low-temperature scanning tunneling microscope (STM) and spectroscopy (STS) provide a direct way to study the SSs through probing the local density of states (LDOS) oscillations in the vicinity of impurities or step edges [16. The quasiparticle interference (QPI) patterns induced by non-magnetic impurities on the surface of $\mathrm{Bi}_{x} \mathrm{Sb}_{1-x}$ [17] and $\mathrm{Bi}_{2} \mathrm{Te}_{3}$ [18, together with subsequent theoretical analysis [19 24], demonstrated the absence of backscattering for the topological SSs. Meanwhile, the LDOS oscillations of SSs near step edges on $\mathrm{Bi}_{2} \mathrm{Te}_{3}$ showed a power-law decay with index -1 in a certain energy range [25], compared to $-1 / 2$ for the conventional two-dimensional electron system (2DES) 16 . The faster decay of QPI once again indicates the suppression of backscattering in TIs.

Despite the intensive investigation, a complete understanding of QPI on the surface of TIs remain elusive partially due to the warping effect of the Dirac cone [8, 26]. The warping effect of the SSs results from not only the anisotropic surface band dispersion, but also the coupling between the surface and bulk bands. In this Letter, we present a general formalism to account for the complex scattering geometry. We propose the interference patterns are dominated by the extremal points on the constant energy contour (CEC) of $2 \mathrm{D}$ electron band. In ap- plying the theory to $\mathrm{Bi}_{2} \mathrm{Te}_{3}$ with strong warping effect, we show that the decay index varies from $-3 / 2$ to $-1 / 2$ and finally to -1 as the energy increases. As for TIs with nearly isotropic Dirac cones, such as $\mathrm{Bi}_{2} \mathrm{Se}_{3}$ [27, the decay index is simply $-3 / 2$. Moreover, the theory can be extended to QPI induced by point defects and readily elucidate the missing of $\vec{q}_{3}$ and the deviation of Fermi velocity in Ref. 18. To confirm the predictions, we have performed STM study on both $\mathrm{Bi}_{2} \mathrm{Te}_{3}$ and $\mathrm{Bi}_{2} \mathrm{Se}_{3}$ films and found excellent agreement with theory. In particular, we have been able to obtain the decay index on $\mathrm{Bi}_{2} \mathrm{Se}_{3}$, whose interference pattern is usually too weak to extract the information.

We start with a general 2D surface band with a single Fermi surface within the surface Brillouin zone (SBZ). Because of elastic scattering, the incoming surface wave with a wave vector $\mathbf{k}^{i}$ must be scattered into the outgoing one with $\mathbf{k}^{f}$ on the same CEC. Assuming a step edge along the $y$-direction, the $k_{y}$ component of the wave vectors should be conserved in a scattering process, i.e. $k_{y}^{i}=k_{y}^{f} \equiv k_{y}$. The interference between the incoming and outgoing waves gives rise to the standing wave oscillation in the $x$-direction. The total LDOS is the sum of contributions from all these oscillations from the SSs on a CEC. For a given energy $E$, we can integrate over $k_{y}$ on the entire CEC and express the LDOS explicitly as

$$
\delta \rho(E, x)=\Re\left[\oint_{E} 2 r /\left(1+|r|^{2}\right) \xi_{i}^{\dagger} \xi_{f} e^{i\left(k_{x}^{f}-k_{x}^{i}\right) x} d k_{y}\right],
$$

where $r$ is the reflection coefficient. Here the scattering wave function is of the form $\xi e^{i k_{x} x+i k_{y} y}$ ( $\xi$ denotes the spin wave function).

A pair of scattering states $\mathbf{k}^{i}$ and $\mathbf{k}^{f}$ lead to a standing wave with a spatial period of $2 \pi /\left(k_{x}^{f}-k_{x}^{i}\right)$. Since the 


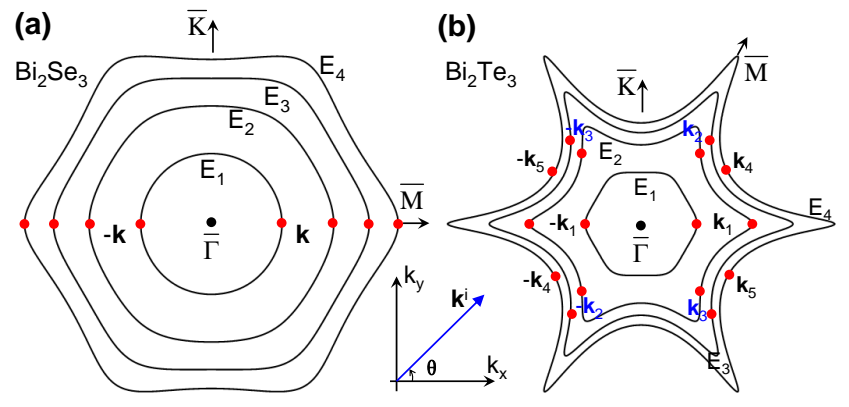

FIG. 1: (color online) Illustration of scattering off the step edge along $y$-direction. The LDOS oscillation is dominated by scattering between the EP pairs on CEC (red dots). (a) The shape of CEC evolves from circle, hexagon to concave hexagon as energy increases in $\mathrm{Bi}_{2} \mathrm{Se}_{3}$. It has a single pair of EPs at $(\mathbf{k},-\mathbf{k})$ and only one type of oscillation pattern appears at different bias voltages. (b) $\mathrm{In} \mathrm{Bi}_{2} \mathrm{Te}_{3}$, as energy increases, CEC evolves from hexagon, concave hexagon to concave hexagram, and the pairs of EPs are first at $\left(\mathbf{k}_{1},-\mathbf{k}_{1}\right)$; then at $\left(\mathbf{k}_{1},-\mathbf{k}_{1}\right),\left(\mathbf{k}_{2},-\mathbf{k}_{3}\right),\left(\mathbf{k}_{3},-\mathbf{k}_{2}\right)$; then at $\left(\mathbf{k}_{2},-\mathbf{k}_{3}\right)$, $\left(\mathbf{k}_{3},-\mathbf{k}_{2}\right)$; finally at $\left(\mathbf{k}_{4},-\mathbf{k}_{5}\right)$ and $\left(\mathbf{k}_{5},-\mathbf{k}_{4}\right)$. Different types of oscillation pattern appear at different bias voltages.

period is different for different value of $k_{y}$, only the pairs whose periods are stationary with respect to small variation in $k_{y}$ can make dominant contribution to the LDOS oscillations. We call such pair of points (with identical $k_{y}$ ) on CEC the extremal points (EPs) 28]. Other standing waves interfere with each other and cancel at large $x$. The spatial dependence of LDOS oscillations in Eq. (1) can be evaluated by expanding the relevant quantities around each EP, namely, let $k_{y}=k_{y 0}+\delta k_{y}$, then $k_{x}^{f}-k_{x}^{i}=\Delta k_{x 0}+\sum_{n} \Delta k_{x n} \delta k_{y}^{n}, r=\sum_{l} \eta_{l} \delta k_{y}^{l}$, and $\xi_{i}^{\dagger} \xi_{f}=\sum_{m} \chi_{m} \delta k_{y}^{m}$. Here $\Delta k_{x 0}$ is the characteristic wave vector depending on the geometry of CEC. To the leading order of $k_{y}$, the LDOS varies at long distance as

$$
\begin{aligned}
\delta \rho(E, x) & \simeq \Re\left[\sum_{\mathrm{EPs}} \int_{E} 2 r /\left(1+|r|^{2}\right) \xi_{i}^{\dagger} \xi_{f} e^{i\left(k_{x}^{f}-k_{x}^{i}\right) x} d k_{y}\right], \\
& \sim \sum_{\mathrm{EPs}}\left|g \eta_{a} \chi_{b} c\right| \cos \left(\Delta k_{x 0} x+\phi_{s}\right) x^{-\frac{a+b+1}{c}},
\end{aligned}
$$

where $a=\min (l), \quad b=\min (m), \quad c=\min (n), \quad$ and $\phi_{s}$ is the initial phase of each pair of EPs. $g$ is given by $\oint_{E} d k^{\prime} k^{\prime(a+b-c+1) / c} e^{i \Delta k_{x c} k^{\prime}}$. The decay behavior of LDOS in Eq. 22 is valid as long as $x \gg \Delta k_{x 0}^{-1}$. The decay index associated with a pair of EPs is given by $(a+b+1) / c$, which is solely determined by the properties of the scattering wave function around the EPs.

Now we turn to the topological SSs on $\mathrm{Bi}_{2} \mathrm{Te}_{3}$ and $\mathrm{Bi}_{2} \mathrm{Se}_{3}$ with a single Dirac cone near $\bar{\Gamma}$ point in the SBZ on each surface. The effective model describing such topological SSs reads [26, 29]

$$
\mathcal{H}(\mathbf{k})=v\left(\sigma_{x} k_{y}-\sigma_{y} k_{x}\right)+\frac{\lambda}{2}\left(k_{+}^{3}+k_{-}^{3}\right) \sigma_{z},
$$

where $\hbar \equiv 1, k_{ \pm} \equiv k_{y} \pm i k_{x}, v$ is the Dirac velocity, $\lambda$ is the warping parameter, and $\sigma_{i}$ are Pauli matrices acting on spin space. For simplicity, here we ignore the particlehole asymmetry as it affects the shape of Fermi surface little. The surface band dispersion is

$$
\varepsilon_{ \pm}\left(k_{x}, k_{y}\right)= \pm \sqrt{v^{2} k^{2}+\lambda^{2} k^{6} \sin ^{2}(3 \theta)},
$$

where $\varepsilon_{ \pm}$denotes respectively the upper and the lower energy bands touching at the Dirac point, and $\mathbf{k} \equiv(k, \theta)$ with $\theta$ as the angle between the wave vector $\mathbf{k}$ and $k_{x^{-}}$ axis $(\bar{\Gamma}-\bar{M})$. The step edge is always along the close packed $\bar{\Gamma}-\bar{K}$ direction. Defining the characteristic energy $\varepsilon^{*} \equiv v \sqrt{v / \lambda}$ and length $\sqrt{\lambda / v}$, in Fig. 1 we plot a set of $\mathrm{CEC}$ of the upper band in momentum space for $\mathrm{Bi}_{2} \mathrm{Se}_{3}$ and $\mathrm{Bi}_{2} \mathrm{Te}_{3}$, respectively. In $\mathrm{Bi}_{2} \mathrm{Se}_{3} \lambda=128 \mathrm{eV} \cdot \AA^{3}$ and $\varepsilon^{*}=0.59 \mathrm{eV}$, so that the CEC is nearly a circle from 0 to $0.42 \varepsilon^{*}(0.25 \mathrm{eV})$ [27]. We plot four representative CEC shown in Fig. 1(a). When the Fermi energy increases, the shape of CEC evolves from a circle $\left(E_{1}=0.31 \varepsilon^{*}\right)$, more hexagon-like $\left(E_{2}=0.55 \varepsilon^{*}\right)$, hexagon $\left(E_{3}=0.7 \varepsilon^{*}\right)$ and to concave hexagon $\left(E_{4}=0.83 \varepsilon^{*}\right)$. In a wide range of energy only a single pair of EPs exists at $(\mathbf{k},-\mathbf{k})$, so the characteristic wave vector is always equal to $2 \mathbf{k}$ and $c=2$. In $\mathrm{Bi}_{2} \mathrm{Te}_{3}$ the warping effect is stronger with $\lambda=250 \mathrm{eV} \cdot \AA^{3}$ and $\varepsilon^{*}=0.23 \mathrm{eV}[\underline{8}$. As shown in Fig. 1(b), EPs evolve with the energy as follows: Single pair of EPs $\left(\mathbf{k}_{1},-\mathbf{k}_{1}\right)$ at $E_{1}=0.7 \varepsilon^{*}$; Multiple pairs of EPs $\left(\mathbf{k}_{1},-\mathbf{k}_{1}\right),\left(\mathbf{k}_{2},-\mathbf{k}_{3}\right)$ and $\left(\mathbf{k}_{3},-\mathbf{k}_{2}\right)$ at $E_{2}=1.46 \varepsilon^{*}>$ $E_{c} \equiv 3^{1 / 3} \sqrt{11 / 9} \varepsilon^{*} \simeq 1.45 \varepsilon^{*}$; Two pairs of EPs $\left(\mathbf{k}_{2},-\mathbf{k}_{3}\right)$ and $\left(\mathbf{k}_{3},-\mathbf{k}_{2}\right)$ survive at $E_{3}=1.91 \varepsilon^{*}$, as the SSs along the $\bar{\Gamma}-\bar{M}$ direction merge into the bulk conduction band; No EPs at all at $E_{4}=2.4 \varepsilon^{*}$, because the SSs separate from bulk one only in the very vicinity along $\bar{\Gamma}-\bar{K}$ direction on the Fermi surface as observed in the ARPES experiment [8]. In this case, scattering between states around $\left(\mathbf{k}_{4},-\mathbf{k}_{5}\right)$ and $\left(\mathbf{k}_{5},-\mathbf{k}_{4}\right)$ will be dominant for LDOS oscillations. Thus in $\mathrm{Bi}_{2} \mathrm{Te}_{3}$ the characteristic wave vector and the LDOS oscillation period critically depend on the bias. In most cases we have parameter $c=2$ except for Fermi energy as high as $E_{4}(c=1)$.

For the incoming state with wave vector $\mathbf{k}^{i}=\left(k^{i}, \theta^{i}\right)$ and energy $\varepsilon_{+}\left(k^{i}\right)$, the inner product of two spin wave functions $\xi_{i}^{\dagger} \xi_{f}=\sin \theta^{i}+i \lambda\left(k^{i}\right)^{3} \sin \left(3 \theta^{i}\right) \cos \theta^{i} / \varepsilon_{+}\left(k^{i}\right)$. It is zero only when $\theta^{i}=0$ as the spins of the time-reversal pairs $(\mathbf{k},-\mathbf{k})$ are orthogonal. Thus, $b=1$ in $\mathrm{Bi}_{2} \mathrm{Se}_{3}$; while in $\mathrm{Bi}_{2} \mathrm{Te}_{3}, b=1$ for the pair $\left(\mathbf{k}_{1},-\mathbf{k}_{1}\right)$, and $b=0$ for other pairs of EPs. Assuming the step edge potential is $V(x)=0$ for $x<0$ and $V(x)=-V_{0}\left(V_{0}>0\right)$ for $x>0$, by matching the boundary condition at the edge the reflection coefficient can be obtained as

$$
r\left(\theta^{i}\right)=\frac{e^{-i\left(\theta^{i}-\theta^{f}\right) / 2}-\beta\left(\theta^{i}\right) e^{i\left(\theta^{i}-\theta^{f}\right) / 2}}{e^{-i\left(\theta^{i}+\theta^{f}\right) / 2}+\beta\left(\theta^{i}\right) e^{i\left(\theta^{i}+\theta^{f}\right) / 2}},
$$

where $\beta\left(\theta^{i}\right)=\left(\varepsilon_{+}\left(k^{i}\right) / k^{i}+\lambda\left(k^{i}\right)^{2} \sin \left(3 \theta^{i}\right)\right) /\left(\varepsilon_{+}\left(k^{f}\right) / k^{f}+\right.$ $\left.\lambda\left(k^{f}\right)^{2} \sin \left(3 \theta^{f}\right)\right), \varepsilon_{+}\left(k^{i}\right)=\varepsilon_{+}\left(k^{f}\right)-V_{0}$ and $\theta^{f}\left(\theta^{i}\right)=$ 
(a)

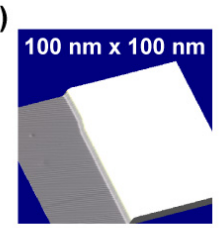

(b)

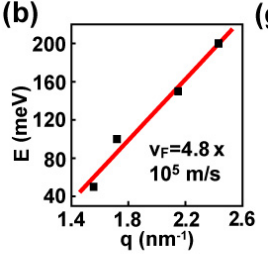

(k)

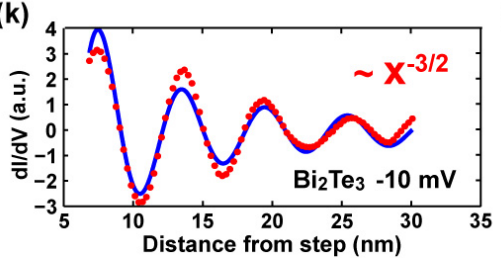

(c)

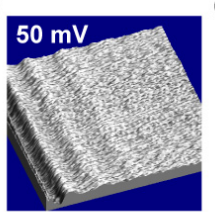

(g)

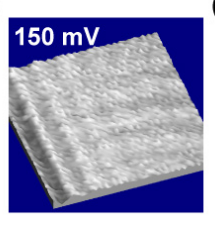

(I)

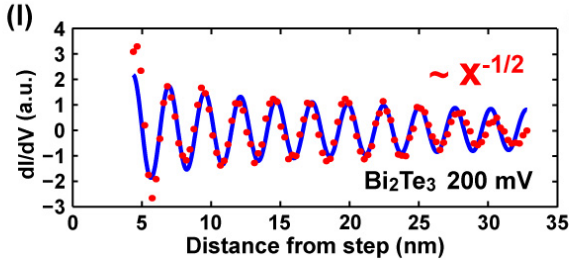

(e)

(i)
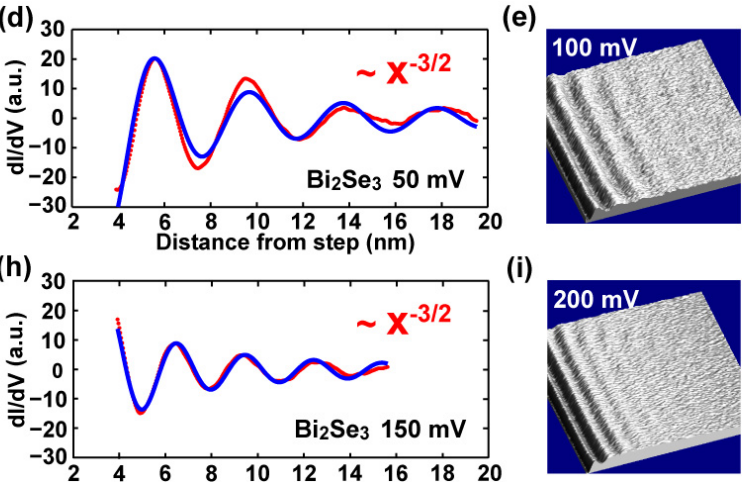

(m)

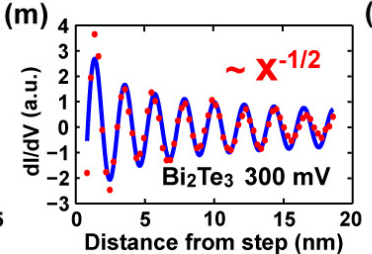

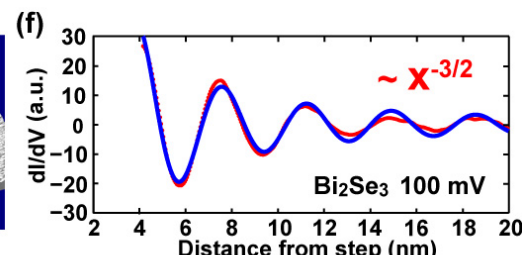

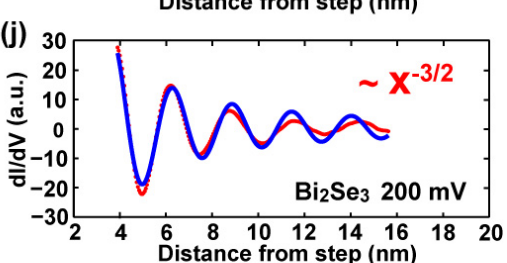

(n)

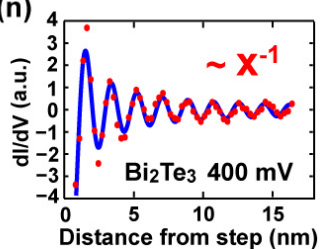

FIG. 2: (color online) LDOS oscillations due to the step edge scattering on the surface of $\mathrm{Bi}_{2} \mathrm{Se}_{3}$ and $\mathrm{Bi}_{2} \mathrm{Te}_{3}$. (a) The $\mathrm{STM}$ topograph of the $\mathrm{Bi}_{2} \mathrm{Se}_{3}(111)$ film, in which a step edge on the surface is observed. (b) Energy dispersion deduced from the standing waves at the step edge. (c)-(j) $d I / d V$ maps and LDOS oscillations for several bias voltages obtained on the upper terrace in film shown in (a). (k)-(n) The LDOS oscillations on $\mathrm{Bi}_{2} \mathrm{Te}_{3}$ at 4 bias voltages. The LDOS is averaged over the width of the step and represented by red dots, and the fitting lines are in blue.

$-\theta^{f}\left(-\theta^{i}\right)$. Due to the constraint by TRS, $r\left(\theta^{i}\right)=$ $-r\left(-\theta^{i}\right)$, and $r\left(\theta^{i}=0\right)=0$. Thus $a=1$ for $(\mathbf{k},-\mathbf{k})$ pair in $\mathrm{Bi}_{2} \mathrm{Se}_{3}$ and $\left(\mathbf{k}_{1},-\mathbf{k}_{1}\right)$ pair in $\mathrm{Bi}_{2} \mathrm{Te}_{3}$, and $a=0$ for other pairs in $\mathrm{Bi}_{2} \mathrm{Te}_{3}$.

In short, the algebraical decay index is $3 / 2$ for $(\mathbf{k},-\mathbf{k})$ and $\left(\mathbf{k}_{1},-\mathbf{k}_{1}\right)$ pairs, $1 / 2$ for $\left(\mathbf{k}_{2},-\mathbf{k}_{3}\right)$ and $\left(\mathbf{k}_{3},-\mathbf{k}_{2}\right)$ pairs, and 1 for $\left(\mathbf{k}_{4},-\mathbf{k}_{5}\right)$ and $\left(\mathbf{k}_{5},-\mathbf{k}_{6}\right)$ pairs. Therefore, the LDOS oscillations of the $\mathrm{SSs}$ in $\mathrm{Bi}_{2} \mathrm{Se}_{3}$ should decay as $x^{-3 / 2}$ in a wide range of energy (as long as $E<0.85 \mathrm{eV}$ ), much faster than $x^{-1 / 2}$ as in $2 \mathrm{DES}\left[16\right.$. On $\mathrm{Bi}_{2} \mathrm{Te}_{3}$ surfaces, as the bias increases, LDOS oscillations decay first as $x^{-3 / 2}(E<0.33 \mathrm{eV})$, then as a combination of $x^{-3 / 2}$ and $x^{-1 / 2}$, then as $x^{-1 / 2}$, and finally $x^{-1}$.

To experimentally confirm the above predictions, we analyzed the interference fringes at the step edges on $\mathrm{Bi}_{2} \mathrm{Se}_{3}$ and $\mathrm{Bi}_{2} \mathrm{Te}_{3}$ surfaces. All experiments were carried out at $4.2 \mathrm{~K}$ in an ultrahigh-vacuum low temperature STM system (Unisoku) equipped with molecular beam epitaxy (MBE) for film growth. The stoichiometric films of $\mathrm{Bi}_{2} \mathrm{Se}_{3}$ and $\mathrm{Bi}_{2} \mathrm{Te}_{3}$ were respectively prepared on graphene and $\mathrm{Si}(111)$ substrates by MBE [30, 31]. A typical STM image of $\mathrm{Bi}_{2} \mathrm{Se}_{3}$ film with a thickness of 50 quintuple layers is shown in Fig. 2(a). We can clearly see the atomically flat morphology of the film and the step of the height of a quintuple layer. The steps are preferentially oriented along the three close packing $(\bar{\Gamma}$ $\bar{K})$ directions. The LDOS of electrons at energy eV is measured through the differential tunneling conductance $d I / d V$ maps by STS. The Fermi velocity along $\bar{\Gamma}-\bar{M}$ is deduced to be $4.8 \times 10^{5} \mathrm{~m} / \mathrm{s}$ by fitting the linear dispersion curve [Fig. 2(b)], in good agreement with the first-principles calculation and the ARPES data [9, 10]. Figs. 2(c)-2(j) exhibit the $d I / d V$ maps on the upper terrace by the step shown in Fig. 2(a) at various bias voltages, and the LDOS as a function of the distance $x$ from the step. The Dirac point is at about $0.18 \mathrm{eV}$ in STS, so the energy of the surface electron counted from the Dirac point in Fig. 2(j) is $0.38 \mathrm{eV}\left(0.64 \varepsilon^{*}\right)$. The best fit to the LDOS oscillations is given by $\delta \rho \propto \cos \left(\Delta k_{x 0} x+\phi\right) x^{-3 / 2}$ as predicted. The suppression of backscattering and the circle-like shape of CEC lead to a much faster decay of LDOS in $\mathrm{Bi}_{2} \mathrm{Se}_{3}$ than that in 2DES [16].

The case of $\mathrm{Bi}_{2} \mathrm{Te}_{3}$ is even more interesting because of the strong warping effect. The LDOS oscillations at 4 different bias voltages on $\mathrm{Bi}_{2} \mathrm{Te}_{3}$ film are shown in Figs. 2 (k)-(n), and the corresponding $d I / d V$ maps were reported in Ref. [18 already. The Dirac point is estimated to be at $-0.21 \mathrm{eV}$ in STS fitting, thus the energies of the surface electron in Figs. 2(k)-2(n) counted from the Dirac point are respectively $0.20 \mathrm{eV}\left(0.87 \varepsilon^{*}\right), 0.41 \mathrm{eV}\left(1.78 \varepsilon^{*}\right)$, $0.51 \mathrm{eV}\left(2.18 \varepsilon^{*}\right), 0.61 \mathrm{eV}\left(2.60 \varepsilon^{*}\right)$. To extract the decay behavior from the LDOS data, for each energy we have compared the fittings with different decay indices. The best fitted LDOS oscillations clearly demonstrate the crossover of the decay from $x^{-3 / 2}$ to a combination of $x^{-3 / 2}$ and $x^{-1 / 2}$, then to $x^{-1 / 2}$, and then to $x^{-1}$ as the bias voltage increases, which agree well with our theoretical predictions. Besides, as predicted theoretically and 


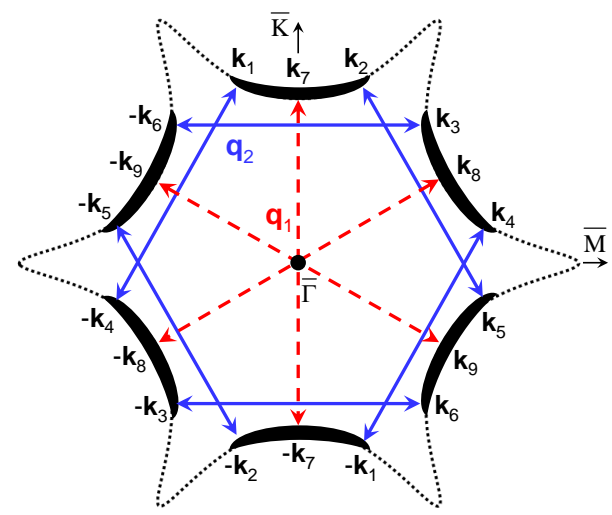

FIG. 3: (color online) The scattering geometry due to a nonmagnetic impurity on $\mathrm{Bi}_{2} \mathrm{Te}_{3}$. When the CEC is a concave hexagram, the high DOS regions are denoted by bold lines along the direction of $\bar{\Gamma}-\bar{K}$. Two kinds of characteristic scattering wave vectors include the forbidden $\vec{q}_{1}$ (dashed arrow) and the allowed $\vec{q}_{2}$ (solid arrow) which connects a pair of EPs.

shown in Figs. 2(1)-2(n), the LDOS oscillates with shorter period as increasing the bias. It is noticeable that due to the strong warping effect of the CEC, the decay rate of LDOS oscillations on $\mathrm{Bi}_{2} \mathrm{Te}_{3}$ is not always faster than in 2DES.

In the last part, we extend our general formalism for the step edge scattering to the non-magnetic impurity scattering on $\mathrm{Bi}_{2} \mathrm{Te}_{3}$. Suppose an incident wave $\psi_{i} \propto e^{i \mathbf{k}^{i} \cdot \mathbf{r}} / \sqrt{r}$ is scattered into $\psi_{f} \propto f(\theta) e^{i \mathbf{k}^{f} \cdot \mathbf{r}} / \sqrt{r}$ by the impurity potential of $U=U_{0} \delta(\mathbf{r})$, then the LDOS oscillates as $\delta \rho \propto f(\theta) e^{i\left(\mathbf{k}^{f}-\mathbf{k}^{i}\right) \cdot \mathbf{r}} / r$ with $f(\theta)$ denoting the scattering amplitude. The characteristic wave vector of the QPI pattern at large distance is obtained when $e^{i\left(\mathbf{k}^{f}-\mathbf{k}^{i}\right) \cdot \mathbf{r}}$ is stationary for certain direction $\hat{r}$. With the concave hexagram Fermi surface of $\mathrm{Bi}_{2} \mathrm{Te}_{3}$ as shown in Fig. 3. there exist only two kinds of characteristic wave vectors: $\vec{q}_{1}$ along $\bar{\Gamma}-\bar{K}$ and $\vec{q}_{2}$ along $\bar{\Gamma}-\bar{M}$ direction. Obviously, the $\vec{q}_{1}$ connects a pair of TRS states whose scattering is forbidden. The $\vec{q}_{2}$ connects a pair of states at EP, which dominates the spatial decay. In such scattering geometry, numerically we find $q_{2}$ vary linearly with the energy and $q_{2}=1.5 \bar{k}$ where $\bar{k}$ is the length of $\bar{\Gamma}-\mathbf{k}_{7}$. Together with the STM data in Rec. 18, we obtain the Dirac velocity along $\bar{\Gamma}-\bar{K}$ as $v=4.15 \times 10^{5} \mathrm{~m} / \mathrm{s}$, in good agreement with ARPES result $\left(v=4.05 \times 10^{5} \mathrm{~m} / \mathrm{s}\right)$ [ $]$. In $\mathrm{Bi}_{2} \mathrm{Se}_{3}$, the CEC is circle-like up to $0.25 \mathrm{eV}$ and the characteristic scattering wave vector is always along the diameter. Therefore, we expect the Fourier transformation of LDOS on $\mathrm{Bi}_{2} \mathrm{Se}_{3}$ surface is ring-like.

In conclusion, our theoretical and experimental investigations indicate that the LDOS oscillation on the surface of TIs is generally determined by the scattering between surface states around the extremal points on Fermi sur- face, either by step edges or by non-magnetic impurities. We have directly observed different standing wave patterns caused by scattering off a step on $\mathrm{Bi}_{2} \mathrm{Te}_{3}$ and $\mathrm{Bi}_{2} \mathrm{Se}_{3}$ with various warped surface bands, which, together with the decay indices at different bias voltages, clearly demonstrate the 2D Dirac nature of topological surface states.

We thank S. C. Zhang, Y. Y. Wang, and R. B. Liu for helpful discussion. This work is supported by the NSFC Grant No. 11074143, and the Program of Basic Research Development of China Grant No. 2011CB921901.

*xc@mail.tsinghua.edu.cn

$\dagger$ bfz@mail.tsinghua.edu.cn

[1] X. L. Qi and S. C. Zhang, Phys. Today 63, No. 1, 33 (2010).

[2] J. E. Moore, Nature 464, 194 (2010).

[3] M. Z. Hasan and C. L. Kane, Rev. Mod. Phys. 82, 3045 (2010).

[4] X. L. Qi and S. C. Zhang, arXiv:1008.2026 [Rev. Mod. Phys. (to be published)].

[5] L. Fu, C. L. Kane, and E. J. Mele, Phys. Rev. Lett. 98, 106803 (2007).

[6] J. E. Moore and L. Balents, Phys. Rev. B 75, 121306 (2007).

[7] D. Hsieh et al., Nature 452, 970 (2008).

[8] Y. L. Chen et al., Science 325, 178 (2009).

[9] H. Zhang et al., Nature Phys. 5, 438 (2009).

[10] Y. Xia et al., et al., Nature Phys. 5, 398 (2009).

[11] D. Hsieh et al., Phys. Rev. Lett. 103, 146401 (2009).

[12] Y. Zhang et al., Nature Phys. 6, 584 (2010).

[13] L. Fu and C. L. Kane, Phys. Rev. Lett. 100, 096407 (2008).

[14] X. L. Qi et al., Phys. Rev. Lett. 102, 187001 (2009).

[15] X. L. Qi et al., Science 323, 1184 (2009).

[16] M. F. Crommie, C. P. Lutz, and D. M. Eigler, Nature 363, 524 (1993).

[17] P. Roushan et al., Nature 460, 1106 (2009).

[18] T. Zhang et al., Phys. Rev. Lett. 103, 266803 (2009).

[19] X. Zhou et al., Phys. Rev. B 80, 245317 (2009).

[20] W. C. Lee et al., Phys. Rev. B 80, 245439 (2009).

[21] Q. H. Wang, D. Wang, and F. C. Zhang, Phys. Rev. B 81, 035104 (2010).

[22] H. M. Guo and M. Franz, Phys. Rev. B 81, 041102 (2010).

[23] R. R. Biswas and A. V. Balatsky, arXiv:1005.4780.

[24] R. R. Biswas and A. V. Balatsky, Phys. Rev. B 83, 075439 (2011).

[25] Z. Alpichshev et al., Phys. Rev. Lett. 104, 016401 (2010).

[26] L. Fu, Phys. Rev. Lett. 103, 266801 (2009).

[27] K. Kuroda et al., Phys. Rev. Lett. 105, 076802 (2010).

[28] L. M. Roth, H. J. Zeiger, and T. A. Kaplan, Phys. Rev. 149, 519 (1966).

[29] C. X. Liu et al., Phys. Rev. B 82, 045122 (2010).

[30] Y. Y. Li et al., Adv. Mater. 22, 4002 (2010).

[31] C. L. Song et al., Appl. Phys. Lett. 97, 143118 (2010). 